\title{
Use of Moonshot Thinking in Space Operational Robotics (SOR) - A Theoretical Analysis
}

\author{
Kawaldeep Kaur \\ Department of Computer \\ Science \& Engg \\ Sri Sai College of \\ Engg and Technology, \\ Mannawala (Amritsar) \\ Punjab (India)
}

\author{
Rajwinder Kaur \\ Department of \\ Computer Science \& Engg1 \\ Sri Sai College of \\ Engg and Technology, \\ Mannawala (Amritsar) \\ Punjab (India)
}

\author{
Jayant Shah Singh \\ Guru Arjan Dev \\ Polytechnic College, \\ Dhariwal (Gurdaspur) \\ Punjab (India)
}

\begin{abstract}
The collection of information from the space is too difficult because of the huge difference in the environment from earth. From studies researchers concluded space operational robotics (SOR) helps a lot for information gathering through space. In this paper, space operational robotics collected information from the space and send to earth for the further use in new inventions. The specific gathered information is real time monitored simultaneously by various devices on the earth by researchers and scientists. This real time monitoring mechanism is followed by using one new approach named as moonshot thinking. The major goal of moonshot thinking is to find out the radical solution without investigating the potential risks will occur in future. This overall scenario is managed with the support of SOR. This SOR complete its operation in two different steps. At first step, SOR collect information from space through intelligent sensors that are embedded on it and in the second step, SOR itself performs mining on collected information while utilizing installed software's on SOR body. The main significance to use the concept of mining on SOR is only sending filtered or mined information that will be definitely useful information on the earth. The additional benefit to use this new designed methodology is it reduces human time, effort, energy and correspondingly save space robotics memory (SRM). Only theoretical analysis has been investigated by authors by considering different data sets of atmospheric conditions with its different attributes. The nature of information received on earth during real time monitoring by space operational robotics is plays very important parameter of this paper.
\end{abstract}

\section{Keywords}

Space operational Robotics (SOR), Moonshot Thinking, Intelligent Sensors, Real Time monitoring device (wireless Camera), Data mining, atmosphere and earth.

\section{INTRODUCTION}

Moonshot Thinking is an edge of tomorrow [35] [37] in engineering field. It is a kind of ground breaking project presented by google whose aim is to provide a radical solution for any global scale problem [25] without studying the profitability and potential risks [20] of anything. The basic idea behind moonshot project is think out of the box [34] and try to solve scalable global problems [25] as like solve for X [22][24][31]. The value of $X$ is undefined. The main significance to use this new approach is to perform maximum reusability operations [26] and achieve multiple better results [29][39] from the scientific studies. In digital world, sometimes researchers are also named this project as a Think tank project [27]. This project can be managed in Google $\mathrm{X}$ labs [22][23] [27][28][30]. And the bigger motivation for launching this think tank project [27] is to improve the overall quality of work [32] for solving global scalable problems [25] that ultimately improves our quality of life [7] transparently by enriching the interactions with the environment [36]. This idea is work more than a technology [33] for us now a days just because of its additional benefits. The practical use of this project is not so simple method but can be easily understand by following three basic steps:

Step-1) Initialize Global Scale Problem [25].

// that is used for solving $X$ [22] [24] [31].

Step-2) Find out the possible Radical Solutions without focusing the near term profitability and its potential risks [31].

// The value of X may be redefine by the researchers that depend on the type of result expectation [40].

Step-3) Finally, Apply Breakthrough technology and collect results [31].

The purpose to utilize this breakthrough technology [18][19] in step 3 is it is operated on low cost servers that ultimately save our cost as well as energy [31]. By following this technology think tank project easily fulfill the major objective of moonshot thinking. As studied by the authors, there are number of projects are handled in google are going now a days as an example project IRIS, project SOLI, project Loon[28] etc but the main focus is only on the think tank project. The reason for that is to save human energy, effort and apply reusability operation for getting more efficient results [29][39]. This reusability operation [26] may or may not be requires some extra effort, extra money as well as extra resources [21]. The practical use of this project is in HP Moonshot [42] [44] function is to allocate cloud data on dedicated servers so that the power maintenance for cloud developers becomes easy [41] and on the other side it will give online instructions to the students for enhancing the level of project learning in the digital environment [43].

This paper presents a human robotics interaction based theoretical analysis in space by utilizing SOR (Space Operational Robotics) [10][38]. HRI follows a criteria having master-slave relationship where human act as a master and robotics act as a slave [1][2][3][4][5]. Tasks can be performed or managed with the collaboration [6] and co-operation $[11][16][17]$ of the environment. The aim of SOR [10] [38] is to capture information from the space and send mined information [9][15] on the earth during real time monitoring. SOR [10] [38] divided its task in two different steps whereas in first step, capture all the information from, the space by utilizing intelligent sensors [8] [14] that already inbuilt in 
SOR body. Here, wireless camera [10][12] is act like an input device in SOR in space. In the second step correspondingly SOR [10][38] own self perform data mining on already captured information from space through embedded software's in SOR body [13] that automatically store the complete record in SOR[10][38] memory. At last, only mined information will be send by wireless camera [10] [12] on earth through real time monitoring device. Most common architecture used by the scientists for real time monitoring can be shown in fig.1:

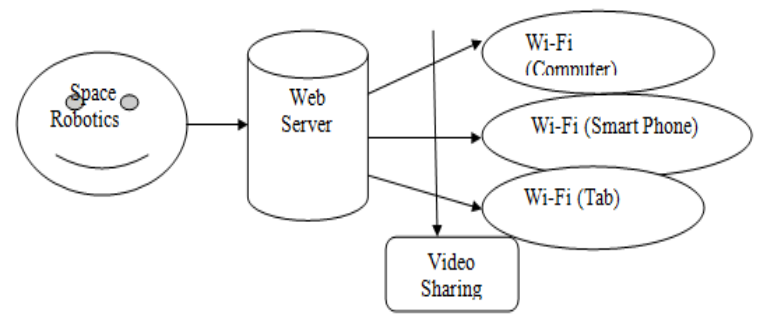

Fig.1: Overview Architecture for: - Remote Monitoring Space Operational Robotics System [9].|

The benefit to utilize this designed methodology is it reduce human effort because of data mining is performed by SOR [10][38] that correspondingly save human time, energy as well as SOR[10][38] memory. On the time of performing theoretical analysis by HRI in space authors considers different data sets of whether forecasting that may either give same or different results that only depend on the type of atmospheric conditions in space on the time of information gathering and can be shown in table.1.

\section{Tadele.: Data set: Different Thether Conditions}

\begin{tabular}{|c|c|c|c|c|}
\hline S.10. & Whether Condition & $\begin{array}{l}\text { Image for } \\
\text { Whether }\end{array}$ & $\begin{array}{l}\text { Temporat } \\
\text { xue Rem } \\
\text { ger }\end{array}$ & Twe ef Information Recievered \\
\hline 1. & $\begin{array}{l}\text { Partly Sumy rith at- } \\
\text { stom }\end{array}$ & & 331027 & Accurate \\
\hline 2. & $\begin{array}{l}\begin{array}{l}\text { Some Sum with } \\
\text { timudestorm }\end{array} \\
\end{array}$ & & 33 to 25 & Approximately Aculuate \\
\hline 3. & Cloudy with a t-stom & & 33 t0 26 & Inaccuate \\
\hline 4. & $\begin{array}{l}\text { Rain and } \\
\text { Thumderstrom }\end{array}$ & & 321028 & Approximately Inacurate \\
\hline 5 & Acid Rain & & Phk6.6. & ERROR \\
\hline
\end{tabular}

The nature of information received on the earth during real time monitoring is an important parameter in this paper.

\section{REVIEW OF LITERATURE}

\section{(Rimmy Chuchra \& Ramandeep Kaur et al Febuary-2013)}

This paper discussed about the joint action taken by the humans and robotics for performing any task. Humans and robotics holds master/slave relationship where interface is act as a sandwich between the humans and robotics. The use of interface is to provide communication between human and robotics in natural way where human acts as a master and is responsible for giving instructions to robotics and robotics always acts as a slave that work as per instructions given by their master. The communication flow methodology between human and robotics can be shown in fig.2: [2]

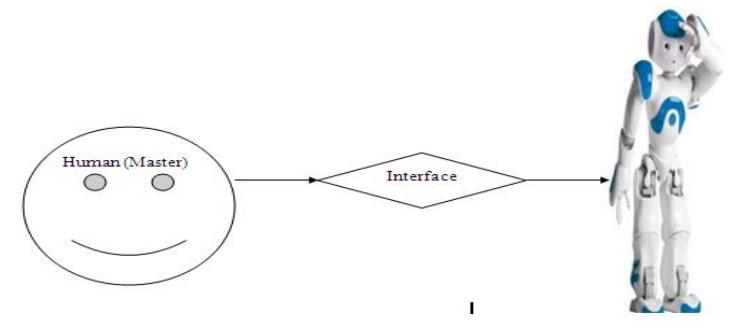

Fig.2: HRI: Shows Master/Slave Relationship.

(Rimmy Chuchra \& R.K Seth et al May-June 2014): Authors had been proposed a new procedure termed as "Human-robotics Interaction based analysis-Using data mining techniques" that shows how humans and robotics performed any task collectively by utilizing three different data mining techniques viz. classification, regression analysis and time series analysis. This designed methodology worked only with single type of data input format. It must be in discontinuous form (i.e. - in digital form). Theoretical and experimental based analysis investigated in this paper with significant results. [1]

(R.K Seth \& Rimmy Chuchra et al January 2015): Authors had designed a new communication flow methodology that works on two separate types of inputs given by the user viz. analog and digital form. The designed methodology also provides more natural way of communication while utilizing interface. Instructions can be easily managed by robotics given by the master-human. [3]

(Rimmy Chuchra \& R.K Seth et al March 2015): This paper presents the synergetic interaction between man and machine having master-slave relationship where a man act as a master and nano-robot act as slave. Here, nano-robot uses sixth sense technology device for mind mapping of idea by utilizing blue brain with swarm intelligence. The major benefit to use sixth sense technology device by nano-robot is to provide protection of human body from the radiations emitted by it (SSTD- as an example smart phone). The collaboration between man and machine intelligence leads to achieve a smart or intelligent joint action for developing projects in any industry or daily life works. [4]

(Rimmy Chuchra \& R.K Seth et al July 2015): This paper presents a qualitative approach for the improvement of existing education system that is termed as "Faculty Performance Metric Scale" (FPMS) by utilizing the application of ambient intelligence. The major goal of this paper is to improve the overall quality of any education system in India by utilizing this designed methodology. The working of FPMS shows synergetic interaction among human and robotics having master-slave relation where human acts as master and robotics act as slave. The main function of service robotics is to store and monitor the faculty members during delivery of lectures. The collaboration between human and service robotics leads to achieve a joint action and shows active team work participation between them (or by utilizing natural interactions). This paper also discusses about the current scenario that are to be followed by on-going universities and after that authors compares the current scenario with the proposed scenario that may help to find out qualitative results in future. [5] 


\section{RESEARCH DESIGN}

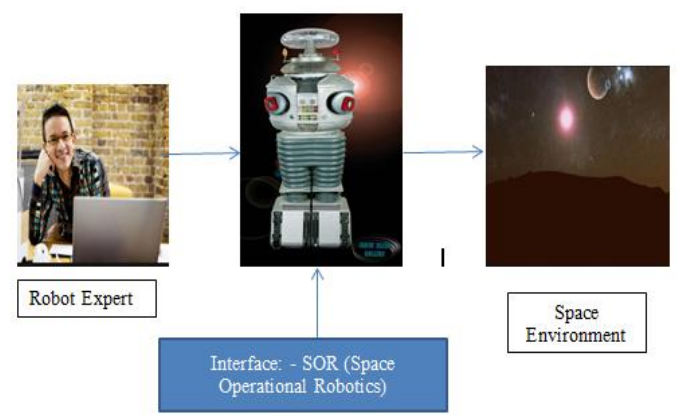

Figure 3: Interaction diagram between robotics expert and space environment through SOR

\subsection{Communication flow diagram between Robotics Expert and Space operational \\ Robotics:}

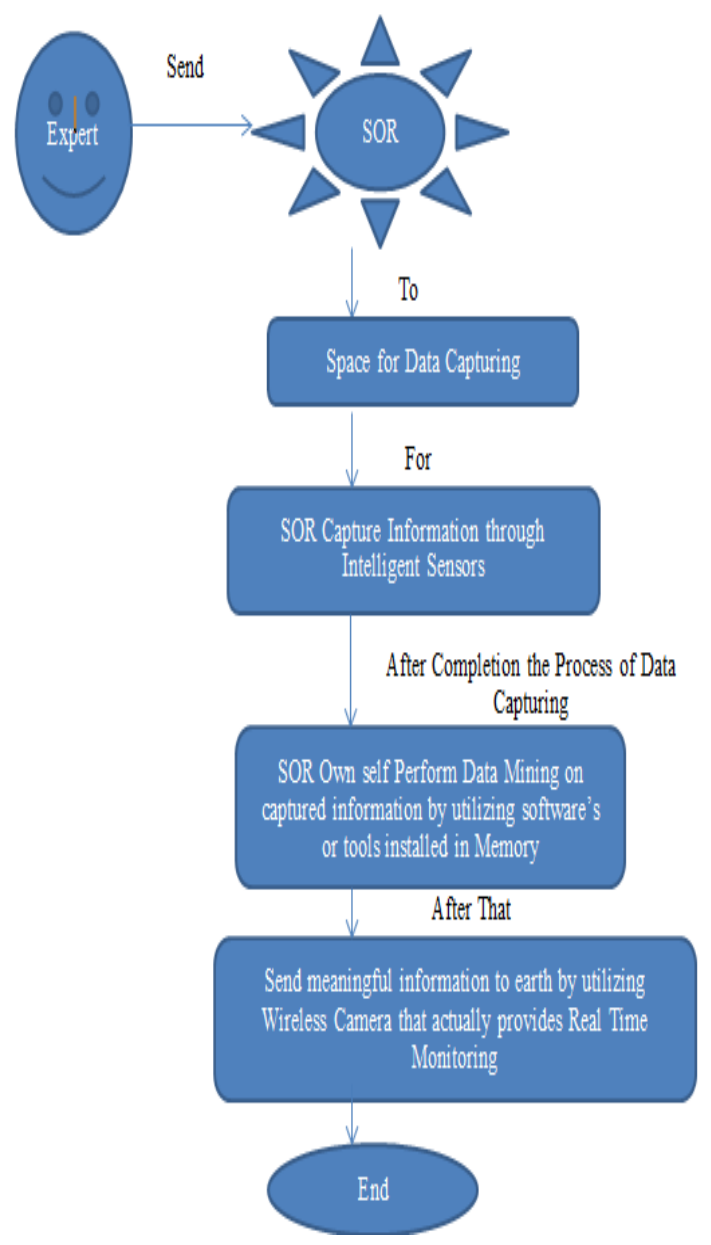

Figure 4: Information Flow Diagram from earth to space.

\section{CONCLUSIONS}

In this paper, human robotics interaction based theoretical analysis in space has been conducted by utilizing moonshot thinking phenomenon. In the absence of interface (i.e. SOR), the interaction between human (so called robotics expert) who is on earth and space will not be possible. This designed methodology may help to provide real time data monitoring during data collection. The most important function of space operational robotics is that only mined information is send on the earth where the separate data sets of whether forecasting are considered in this paper. Different data sets may help us to receive nature of information on earth as an example received information gives accurate results, approximately accurate, inaccurate, approximately inaccurate or error message. Authors considered error message in exceptional case it will only come when space the weather condition is unpredictable or undefined which is very rare. A variety of algorithms, scientific tools and techniques can be programmed into SOR by robotics expert.

\section{FUTURE SCOPE}

This work will be extended by inserting blue brain/virtual brain of any scientist say albert Einstein into space operational robotics (SOR). The significance to utilize this blue brain project in artificial intelligence is data capturing from space on the basis of past knowledge and experiences. The chances to collect useful information from space will be increases because of it will may work for researchers little bit more than artificial intelligence (i.e. more than artificial intelligence but less than actual/active human intelligence) and correspondingly it also fulfill the main goal of moonshot thinking that is Reusability operation. In this way, the reusability of Albert Einstein brain may increase s to capture the chances of meaningful information from space through his intelligence and past experiences.

\section{REFERENCES}

[1] Rimmy Chuchra \& R.K Seth, March-2014. Human robotics interaction based analysis-Using DMT, International Journal of Computer Science and Information Technology.

[2] Rimmy chuchra \& Ramandeep kaur, Febuary 2013. Human robotics interaction with data mining techniques", International Journal of Emerging Technology And Advance Engg.

[3] R.K Seth \& Rimmy Chuchra, January 2015. Synergetic Interaction among Humans and Robotics By Proposing Communication Flow Methodology, International Journal of Computer Applications, New York.

[4] Rimmy Chuchra \& R.K Seth, March-2015. MIII: Proposing a Communication Flow Methodology for improving collaborative learning in A.I, International Journal of Computer Applications, New York.

[5] Rimmy Chuchra \& R.K Seth, July-2015. FPMS: A Qualitative approach Using Ambient Intelligence, International Journal of Computer Applications, New York.

[6] Baoye Song,Yinghua Xue,Fengyu Zhou,Guohui Tian and Feilu, 2012.Building an Intelligent home space for service robot based on multi pattern Information Model and wireless sensor network, Scientific research,Intelligent Control and automation.

[7] Henrik I. Christensen and Peter Allen, June 2015. Toward a science and autonomy for physical systems: Service, Computing Communication Consortium.

[8] Mumin SONG, Zhiyong LIU, Guohui TIAN and Fei LU, 2014.An Automatic approach of service robot based on parameter Driven Mechanism, Journal of computational Information Systems, China 
[9] Yong-Ho Seo \& Taewoo Han, 2014. Development of a service Robot system for a remote Child Monitoring Platform, International journal of Smart Home.

[10] Prof.S.N Longani, Prof.T.S Ready Shital Shelar, Sanket Gailkwad and Jacksen Alex, 2015. Space and Geographical Operational Robot, International Research Journal of Innovative Engg.

[11] Jurgen Leitner, A Survey of Multi-Robot Co-operation in Space, Intelligent Space Systems Laboratory, Japan.

[12] Kohtaro Ohba, Bong Keung Kim and Sidiq S.Hidayat, 2012.An approach for robots to deal with objects, International Journal of computer Science and Information Technology.

[13] Ningyang Wang,Yinffeng Chen,Dongcai Lui,Xiaopin Chen, 2014.The intelligent Service Robot for Robocop@ home 2014

[14] Demetri Terzopoulos and Faisal Quresh, 2007. Intelligent Perception and Control for Space Robotics ,Machine Vision and Applications, Springer-Verleg.

[15] Migbar Assefa, 2013.Strategies for control of space robots: A Review and research agenda, Global Journal of researches in Engg Mechanical and Mechanics Engg.

[16] L.Nourbaksh, D.Wettergreen, D.Kortenkamp and Pedersen, A Survey of Space Robotics, QSS Group,Inc at NASA ARC.

[17] John Schreiner,Lorenzo Fluckiger,Clayton Kunz,Illah Nourbaksh,Terrence Fong, 2005. The peer-to-peer HRI Project,National Institute of standards and technology, Space ,California.

[18] Edward W.Tunstel and Ayanna M.Howard,2006. Intelligent for space robotics, NASA Jet Propulsion Laborator,TSI Press.

[19] S.Kaff, P.Staritz, Wetter-Green, S.Singh, New Themes in Robotics Exploration and assembly for space applications, Field Robotics Centre.

[20] Whatis.techtarget.com/definition/moonshot.

[21] www.wired.com/2013/02/moonshots-matter-heres-howto-make-them-happen.

[22] branchannel.com/2015/03/04/how-moonshot-thinkinginform-everything-google-does/
[23] fitplan.io/10X-moonshot-thinking/.

[24] Googleblog.blogspot.in/2013/o2/solve-for-x-celebratingmoonshot.html.

[25] www.huffingtonpost.Com/2012/02/06/google-unveilssolve-for-n-1258870.html?ir=india\&adsSiteOverride=in .

[26] www.pcmag.com/article/0,2817,2453259,00.asp.

[27] https://en.wikipedia.org/wiki/solve-for-X.

[28] Kernelmag.dailyelot.com/issue-sections/features-issuesections/12083/google-x-project-loon-titan-makhani.

[29] techchomp.blogspot.in/2013/04/moonshot-thinking.html.

[30] Schdule.sxsw.com/2013/events-IAP993258.

[31] blog.aha.io/index.php/should-your-startup-take-amoonshot/.

[32] moonshotdigital.com/how-we-work.

[33] wscannestastic.tumblr.com/Post/53338240093/lessonsfrom-google-on-moonshot-thinking-it-is.

[34] www.pocket.lint.com/news/12668/-google-x-explainedwhat-s-really-going-on-at-google-s-secret-lab.

[35] sukshma.net/2014/10/04/moonshot-thinking-the-filmthe-edge-of-tomorrow/.

[36] healthxl.org/what-are-healthcare-moonshots/.

[37] www.voanews.com/content(futuristic-robots-come-tolife-in-virginia-tech-lab-111387534/163436.html.

[38] https://en.wikipedia.org/wiki/future-of-robotics.

[39] www.slideshare.net/fidaushkan10/iicc2013-fk-googlemoonshots.

[40] www.compudynamics.co.za/whats-your-x-amplifyingtechnology-moonshots.

[41] Cloudtimes.org/2013/06/19/hp-launches-new-productscloud-OS-and-the-moonshot/.

[42] blog.cue.org/moonshots-in-education/.

[43] stevenmorrane.blogspot.in/2014/10/moonshotthinking.html.

[44] R.Buckminster Fuller,'The clean Energy Moonshot-A California Pilot Project,World Business Acadmy or//Worldbusiness.org/clean-energy. 